\title{
Phytophthora Database 2.0: Update and Future Direction
}

\author{
Bongsoo Park, Frank Martin, David M. Geiser, Hye-Seon Kim, Michele A. Mansfield, Ekaterina Nikolaeva, \\ Sook-Young Park, Michael D. Coffey, Joseph Russo, Seong H. Kim, Yilmaz Balci, Gloria Abad, Treena Burgess, \\ Niklaus J. Grünwald, Kyeongchae Cheong, Jaeyoung Choi, Yong-Hwan Lee, and Seogchan Kang
}

First, third, and eighteenth authors: Integrative Biosciences Graduate Program in Bioinformatics and Genomics, and third, fourth, fifth, sixth, seventh, and eighteenth authors: Department of Plant Pathology \& Environmental Microbiology, Penn State, University Park, PA 16802; second author: USDA-ARS Crop Improvement and Protection Research Unit, Salinas, CA 93906; eighth author: Department of Plant Pathology \& Microbiology, University of California, Riverside, CA 92521; ninth author: ZedX, Inc., Bellefonte, PA 16823; tenth author: Pennsylvania Department of Agriculture, Harrisburg, PA 17110; eleventh author: Department of Plant Science \& Landscape Architecture, University of Maryland, College Park, MD 20742; twelfth author: USDA-APHIS-PPQ, Beltsville, MD 20705; thirteenth author: Center for Phytophthora Science and Management, Murdoch University, Australia; fourteenth author: USDA-ARS Horticultural Crops Research Laboratory, Corvallis, OR 97330; and fifteenth, sixteenth, and seventeenth authors: Department of Agricultural Biotechnology and Center for Fungal Pathogenesis, Seoul National University, Seoul 151-921, Korea.

Accepted for publication 12 June 2013.

\section{ABSTRACT}

Park, B., Martin, F., Geiser, D. M., Kim, H.-S., Mansfield, M. A., Nikolaeva, E., Park, S.-Y., Coffey, M. D., Russo, J., Kim, S. H., Balci, Y., Abad, G., Burgess, T., Grünwald, N. J., Cheong, K., Choi, J., Lee, Y.-H., and Kang, S. 2013. Phytophthora database 2.0: Update and future direction. Phytopathology 103:1204-1208.

The online community resource Phytophthora database (PD) was developed to support accurate and rapid identification of Phytophthora and to help characterize and catalog the diversity and evolutionary relationships within the genus. Since its release in 2008, the sequence database has grown to cover 1 to 12 loci for $\approx 2,600$ isolates (representing 138 described and provisional species). Sequences of multiple mitochondrial loci were added to complement nuclear loci-based phylogenetic analyses and diagnostic tool development. Key characteristics of most newly described and provisional species have been summarized. Other additions to improve the PD functionality include: (i) geographic information system tools that enable users to visualize the geographic origins of chosen isolates on a global-scale map, (ii) a tool for comparing genetic similarity between isolates via microsatellite markers to support population genetic studies, (iii) a comprehensive review of molecular diagnostics tools and relevant references, (iv) sequence alignments used to develop polymerase chain reaction-based diagnostics tools to support their utilization and new diagnostic tool development, and (v) an online community forum for sharing and preserving experience and knowledge accumulated in the global Phytophthora community. Here we present how these improvements can support users and discuss the PD's future direction.

Additional keywords: culture collection, database, invasive pathogen, molecular diagnosis, population biology, systematics.
Why was the Phytophthora database developed? Few pathogen groups can claim the notoriety of the genus Phytophthora, which includes old nemeses like $P$. infestans and $P$. capsici that continuously threaten agricultural systems, and recently emerged novel pathogens of global concern, such as $P$. ramorum and $P$. kernoviae $(2,13)$. Due in large part to increased surveys of agricultural and natural ecosystems in recent years, many novel species, including those that appear to be interspecies hybrids, have been discovered $(2,4,8,12,13)$. However, despite this advancement, the large estimated diversity of Phytophthora underscores that our understanding of its ecology and diversity is still limited $(2,8,13)$. Not all novel species will pose a major threat to crop production and/or the environment, but if history is a guide, some will. To effectively support rapid and accurate identification of pathogens, a key step for disease prevention and management,

Corresponding authors: F. Martin; E-mail address: Frank.Martin@ars.usda.gov and S. Kang; E-mail address: sxk55@psu.edu

B. Park, F. Martin, and S. Kang contributed equally to this work.

* The $e$-Xtra logo stands for "electronic extra" and indicates that Figures 1 and 2 appear in color online.

http://dx.doi.org/10.1094/PHYTO-01-13-0023-R

(C) 2013 The American Phytopathological Society we cannot overemphasize the importance of cataloging cultures and associated data that represent the known diversity of the genus as references in a readily accessible format. Because Phytophthora pathogens pose a global threat, establishing a mechanism that facilitates international cooperation in documenting the diversity and dynamics of known and novel pathogens also is extremely critical $(10,11)$. To address these needs, the Phytophthora database (PD; http://www.phytophthoradb.org/) was developed (14).

The first version consisted of the following components: (i) databases that archived genotypic and phenotypic data associated with individual species and isolates, (ii) tools for searching and analyzing archived data, and (iii) user interfaces for data browsing, storage, and submission (14). To support quick identification of newly isolated Phytophthora strains via comparison of sequences from phylogenetically informative loci with the corresponding sequences derived from known species, as well as subsequent phylogenetic analyses for potentially new species, the sequence database was populated with multilocus data from 82 species that were represented by $\approx 1,500$ isolates $(1,14)$. The species page contained an array of information, such as a genuswide phylogenetic tree, nomenclature, morphological and growth characteristics, hosts and disease symptoms, life cycle, and selected references, to help users quickly learn about key characteristics of each species. Since the publication of the PD in 2008 (14), many new data, information, and tools have been incorporated to ex- 
pand its utility and functionality, and its user interface has gone through multiple rounds of modification (Fig. 1).

The genotypic database has grown in the amount and type of data. The most frequent use of the PD is identifying new isolates using its sequence database, which has contributed to exploring the diversity of Phytophthora. To support accurate and robust identification, its sequence database has been updated regularly by depositing data generated from newly described species and correcting historical errors associated with certain isolates. When available, we also included data from provisional species to help prevent duplicated description of new species. The sequence database currently archives sequences of 1 to 12 loci from $\approx 2,800$ isolates (representing 117 species and 21 provisionally described species). We replaced the phylogenetic tree on the species page with a new tree based on sequences of four mitochondrial loci (coxl, nad9, rps10, and secY). The new tree includes the original 82 species (1) plus 24 species that have been formally described or provisionally identified since 2008 (F. Martin, J. Blair, and M. Coffey, in preparation); comparisons of these trees exhibited limited differences in evolutionary relationships between nuclear and mitochondrial loci. As the Barcode of Life Database project (16) has been mainly based on the coxl gene, data for this locus was also added. These mitochondrial loci are easily amplified under standard polymerase chain reaction (PCR) conditions and should complement phylogenetic analyses and diagnostic tools based on nuclear loci. Primers and PCR conditions for amplifying these and nuclear markers are described in the Genetic Markers section under the "Database" menu.

With the exception of a core set of several hundred isolates used for the phylogenetic analyses described above, only sequences of the internal transcribed spacer (ITS) region and to a lesser degree cox 2 have been generated from most other isolates. Some of these isolates were probably misidentified when they were deposited to culture collections, and the sequence data generated from such isolates became associated with incorrect species information. To correct such historical errors, the ITS and cox2 sequence data were examined. First, all ITS sequences were aligned based on their species annotation. Sequences that exhibited a high number of polymorphic sites to those from other strains within the same species were used to run BLAST searches against the PD sequence database. Those that showed much higher identity to ITS sequences from other species than to the annotated species designation were considered misidentified when the strains were deposited to culture collection. When available, $\operatorname{cox} 2$ data were also examined to confirm the results obtained from the ITS region. Although this data curation process was not sufficient to eliminate all errors, all obvious misclassifications were corrected. To ensure the accuracy of species identification with future sequence deposits, prior to uploading the new data they will be compared against a sequence database of standard isolates representing all species in the genus. Any sequences with a questionable species classification will be re-
Sequence alignment
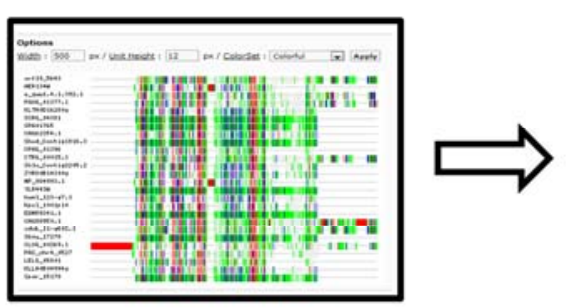

Phylogenetic analysis
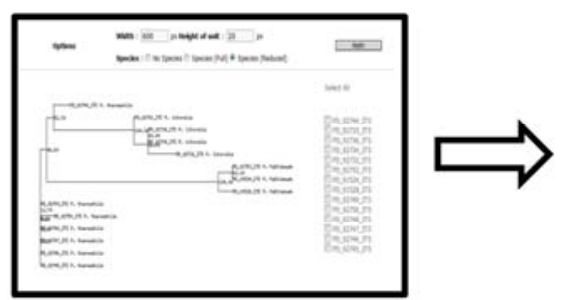

\section{Personalized data management}

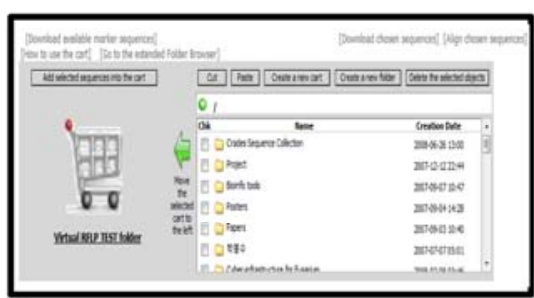

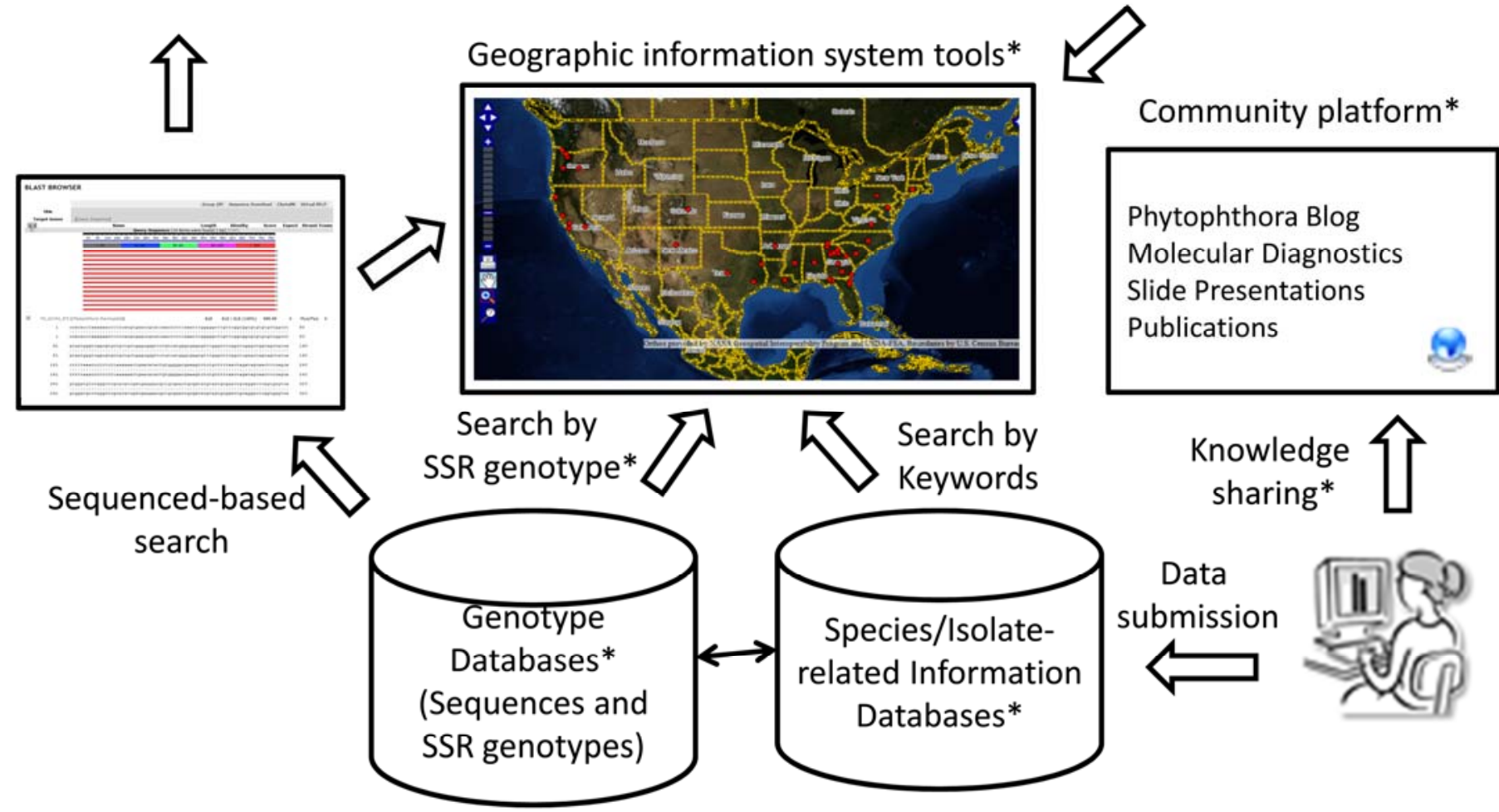

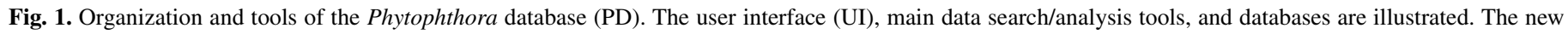

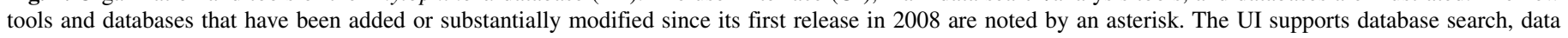
submission, and navigation through the PD and has been updated multiple times. 
checked against sequences from all isolates of the indicated species to assess if differences are due to intraspecific variation or if the isolate needs to be rechecked to correct the species classification.

Microsatellites (simple sequence repeat) have been widely used to study the evolutionary processes within species and to infer their putative migration patterns, helping to understand potential mechanisms underpinning changes in pathogen communities (7). To archive and disseminate results from such studies, we created a database for microsatellite markers. In the current release we provided multilocus data for $P$. ramorum from several recent studies $(5,6,9,15,17)$. This new database was designed to archive data from other species when researchers wish to deposit their results. A tool for comparing multilocus microsatellite genotypes was developed to support searches of this new database. To initiate a search, users select the genotypes of a query strain at each of the characterized loci via a pull-down menu that shows all known alleles. This tool also allows users to search the archived isolates based on host of origin, geographic origin, and mating type (Fig. 2). The search result is displayed in a tabular format and includes the strain accession number, similarity score, and matching loci. Geographic origins of any isolates on the table can be viewed on a global map by clicking the 'Display the chosen isolates on a map' button.

Geographic information system (GIS) tools enable the visualization of geographic origins of archived isolates. These tools (Fig. 2) are based on ortho-imagery from the National Aeronautics and Space Administration's Geospatial Interoperability Program and the USDA Farm Service Agency. The map with a zoom function can be overlaid with reference data layers, including political boundaries, rivers, roads, and railroads from the
United States Census Bureau's Topologically Integrated Geographic Encoding and Referencing (TIGER) data set. A query tool allows users to display all Phytophthora isolates collected at a given geographic location with links to isolate information in the isolate database. The GIS tools were incorporated into a number of places (e.g., the species page, BLAST results, and microsatellite search) to allow users to visualize the distribution of chosen isolates (Fig. 1).

A step-by-step user guide for how to use the GIS tools was incorporated. The capability of visualizing geographic origins of individual species and isolates helps understand the geospatial structure and distribution of Phytophthora, providing clues to the likely origin of a new isolate. In combination with microsatellite data, this new tool set helps understand the population structure and dynamics in geospatial context. Unfortunately, many historical isolates in the PD lack detailed information on their geographic origin; typically information ends at the country or state/ province level. This currently limits the utility of the GIS tools, but most cultures resulted from recent surveys are attached to global positioning system (GPS) coordinates.

Additional changes also expanded the functionality of the PD. The "Molecular Diagnostics" section was created to provide an overview of the following molecular diagnostics techniques used with Phytophthora: (i) identification via DNA sequencing, (ii) gel-based identification of species, (iii) genus-specific diagnostic markers, (iv) species-specific diagnostic markers, (v) identification of subpopulations, and (vi) macro/micro-arrays. A brief description of each method and its applications with key references is provided. Sequence alignments of the major loci used in PCR marker design are available for downloading so that users can quickly build their own markers. Given that the most

\section{A}

\begin{tabular}{ll} 
1. Search by isolate informatio \\
\hline HOST & Select the H \\
STATE & California \\
COUNTRY & United Stat \\
MATING TYPE & Unknown \\
& \\
& \\
2. Search by SSR genotype \\
\multicolumn{2}{l}{ SSR Markers }
\end{tabular}

\begin{tabular}{l|ll|} 
PrMS6 & $165 / 165$ & \\
\cline { 2 - 3 } Pr9C3 & $218 / 224$ & \\
PrMS39 & $136 / 140$ & \\
PrMS45 & $166 / 186$ & \\
PrMS43 & $134 / 145$ & \\
18 & $217 / 261$ & \\
18 & & \\
64 & $350 / 401$ & \\
\hline
\end{tabular}

\section{New alleles $(X / Y)$}

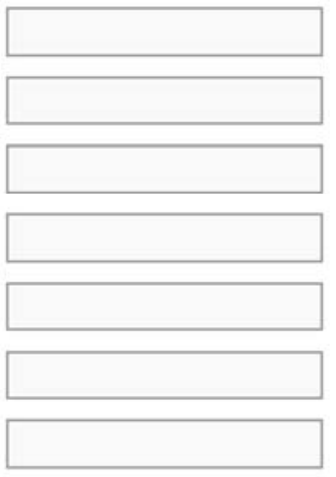

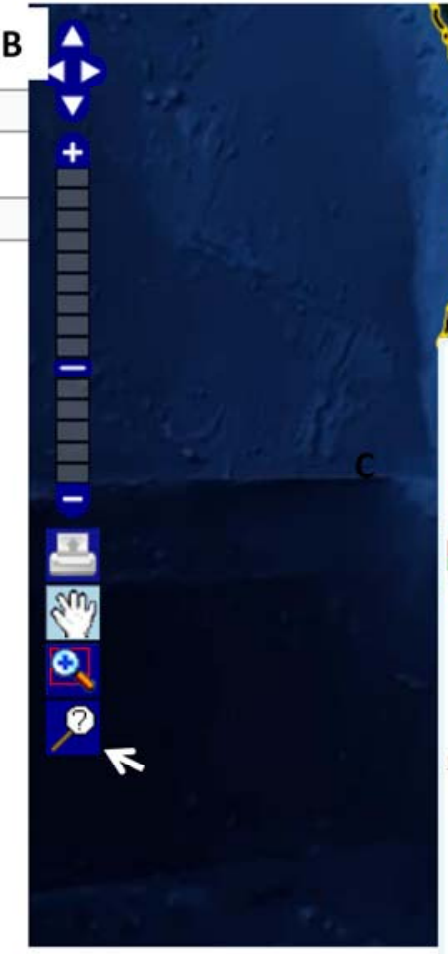

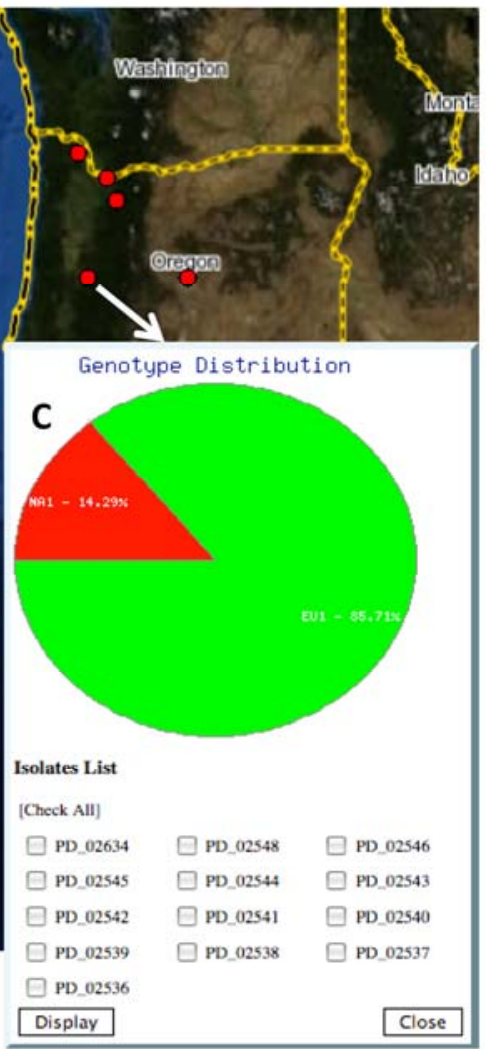

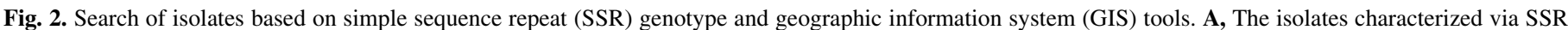

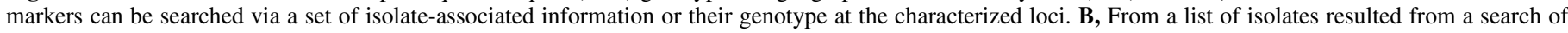

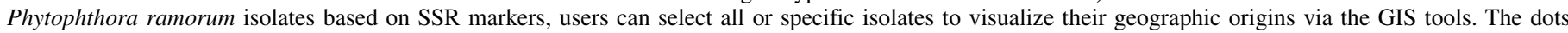

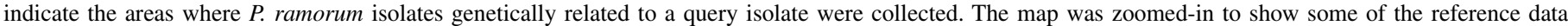

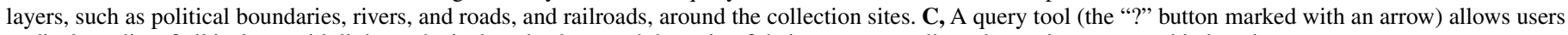
to display a list of all isolates with links to the isolate database and the ratio of their genotype collected at a given geographic location. 
frequently used function is BLAST, which allows users to identify a new isolate via its sequence similarity to those archived in the PD, the BLAST interface is now shown at the home page so that users do not need to open it through the "Search \& Analysis" menu. A graphic overview of the list of loci that have been sequenced and the number of sequences from each locus is shown at the home page, which gets automatically updated, and is linked to corresponding databases.

To effectively curate educational materials and to encourage contribution of new materials by PD users, a new tool entitled Community Platform (CP) was created. The content and tools in this platform are organized in the following three sections: (i) Phytophthora Blog, (ii) Slide Presentations, and (iii) Publications. The main function of "Phytophthora Blog" is to provide an online forum for sharing knowledge and experience with others in the community via its blog interface. The trail of communications associated with the archived posts will help new comers to quickly learn from the accumulated collective knowledge and experience rather than learning them through trial and error. This social media function will also serve as an ideal conduit for rapidly sharing information on emerging disease problems and function to support global networking of those who study Phytophthora. The "Slide Presentations" section currently holds presentations given at two international workshops on Phytophthora to make the information available to a broader audience. The "Publications" section provides a list of all Phytophthorarelated papers archived in PubMed.

Continued growth of the PD heavily depends on community participation. The number of registered PD users is 640 from $\approx 50$ countries with $\approx 2,600$ visits per month. Through the updates described here, the utility and functionality of the PD has increased. However, the PD continues to remain as a work in progress and requires suggestions for improvement and contribution of content by the global Phytophthora research community. Similar to many other online community resources, its long-term sustainability and growth depends heavily on contributions from the community it serves. With the wide adoption of molecular evolutionary principles for species recognition for fungi and oomycetes (3), a key area that requires contribution from the global Phytophthora community is the collective curation and update of the genus-wide phylogenetic data. Without such data sharing and curation, community efforts to discover and describe novel species will likely be fragmented and redundant, creating confusion instead of the order that taxonomy should provide. Curated phylogenetic data in the PD will also facilitate the development of robust sequence-based detection and identification methods and the recognition of historical errors in strain identification and questionable species boundaries. Phylogenetic analyses suggest that several traditionally defined species such as $P$. capsici, $P$. citricola, $P$. citrophthora, $P$. cryptogea, and $P$. drechsleri are complexes consisting of several cryptic species, and reexamination of culture collections by sequencing also has indicated that potentially novel species remain misidentified, waiting for reevaluation $(2,11)$.

We envision that the information content available in $\mathrm{CP}$ will continue to grow as a depository for technical experience and knowledge (e.g., protocols and tips) in the global Phytophthora community. Since the advancement of science heavily relies on how effectively we build on existing knowledge and experiences, it is critical to establish an efficient mechanism to archive and share such resources. This type of knowledge archiving is critical to effectively leverage new knowledge and respond to Phytophthora threats based on the best available information and also assist new generations of researchers and educators in quickly learning from the collective knowledge and experience. Social media technology offers immense potential for supporting and coordinating this type of activity without being limited by distance and traditional forms of organizational structure.
Although DNA sequences and molecular tools are routinely utilized for diagnosis, they cannot completely replace traditional diagnostic approaches based on morphological and growth characteristics. A summary of key phenotypical characteristics of individual species via the species page of the PD is not designed to provide such support. To address this need, the PD will be linked to a new web resource, entitled "Online Identification Tools for Phytophthora with Interactive Lucid Key, Tabular Key and Sequencing Analysis," currently under development (expected to be released in 2014). This new diagnostics resource will provide morphological data derived from authenticated species in two different formats (G. Abad and Y. Balci, unpublished data). The Lucid Key (based on Lucid v. 3.5) is a matrix-based identification tool based on a set of 22 morphological features (e.g., sporangium, oogonium, chlamydospore, and hyphal swelling) and 92 character states (e.g., sporangium papillate, semipapillate, and nonpapillate). The Tabular Key (13) helps users to quickly narrow down the likely identity of a new isolate using morphological features and design additional observations to confirm its identity.

\section{ACKNOWLEDGMENTS}

We thank the many individuals who contributed data to the PD. Their contribution was acknowledged at the home page or the species page. Grants from the USDA-AFRI Plant Biosecurity program supported the establishment and improvement of the PD (2005-35605-15393 and 200855605-18773). Establishment of the PD was also supported by grants from the National Research Foundation of Korea (2012-0001149 and 2012-0000141) and the Next-Generation BioGreen 21 Program of Rural Development Administration in Korea (PJ00821201) to Y.-H. Lee. Support from the Pennsylvania Department of Agriculture (ME 445580, 44091394, and 44102197) has also contributed to generating data and updating the PD. S. Kang and B. Park acknowledge fellowship support from the Penn State Institute for Cyber-Science.

\section{LITERATURE CITED}

1. Blair, J. E., Coffey, M. D., Park, S.-Y., Geiser, D. M., and Kang, S. 2007. A multi-locus phylogeny for Phytophthora utilizing markers derived from complete pathogen genomes. Fungal Genet. Biol. 45:266-277.

2. Brasier, C. 2009. Phytophthora biodiversity: How many Phytophthora species are there? Pages 101-115 in: Phytophthoras in Forests and Natural Ecosystems. E. Goheen and S. Frankel, eds. General Technical Report PSW-GTR-221, USDA Forest Service, Albany, CA

3. Geiser, D. M. 2004. Practical fungal molecular taxonomy. Pages 1-12 in: Advances in Fungal Biotechnology for Industry. J. Tkacz and L. Lange, eds. Kluwer Academic Publishers, Dordrecht, The Netherlands.

4. Goss, E. M., Cardenas, M. E., Myers, K., Forbes, G. A., Fry, W. E., Restrepo, S., and Grünwald, N. J. 2011. The plant pathogen Phytophthora andina emerged via hybridization of an unknown Phytophthora species and the irish potato famine pathogen, P. infestans. PLoS One 6:e24543.

5. Goss, E. M., Larsen, M., Chastagner, G. A., Givens, D. R., and Grünwald N. J. 2009. Population genetic analysis infers migration pathways of Phytophthora ramorum in US nurseries. PLoS Pathogen 5:e1000583.

6. Goss, E. M., Larsen, M., Vercauteren, A., Werres, S., Heungens, K., and Grünwald, N. J. 2011. Phytophthora ramorum in Canada: Evidence for migration within North America and from Europe. Phytopathology 101:166-171.

7. Grünwald, N. J., and Goss, E. M. 2011. Evolution and population genetics of exotic and re-emerging pathogens: Novel tools and approaches. Annu. Rev. Phytopathol. 49:249-267.

8. Hansen, E. M., Reeser, P. W., and Sutton, W. 2012. Phytophthora beyond agriculture. Annu. Rev. Phytopathol. 50:359-378.

9. Ivors, K., Garbelotto, M., Vries, I. D., Ruyter-Spira, C., Te Hekkert, B., Rosenzweig, N., and Bonants, P. 2006. Microsatellite markers identify three lineages of Phytophthora ramorum in US nurseries, yet single lineages in US forest and European nursery populations. Mol. Ecol. 15:1493-1505

10. Kang, S., Blair, J. E., Geiser, D. M., Khang, C. H., Park, S.-Y., Gahegan, M., O'Donnell, K., Luster, D. G., Kim, S. H., Ivors, K. L., Lee, Y.-H., Lee, Y.-W., Grünwald, N. J., Martin, F. M., Coffey, M. D., Veeraraghavan, N., and Makalowska, I. 2006. Plant pathogen culture collections: It takes a village to preserve these resources vital to the advancement of agricultural security and plant pathology. Phytopathology 96:920-925. 
11. Kang, S., Mansfield, M. A., Park, B., Geiser, D. M., Ivors, K. L., Coffey, M. D., Grünwald, N. J., Martin, F. N., Lévesque, C. A., and Blair, J. E. 2010. The promise and pitfalls of sequence-based identification of plant pathogenic fungi and oomycetes. Phytopathology 100:732-737.

12. Kroon, L. P. N. M., Brouwer, H., de Cock, A. W. A. M., and Govers, F. 2012. The genus Phytophthora Anno 2012. Phytopathology 102:348-364.

13. Martin, F. N., Abad, Z. G., Balci, Y., and Ivors, K. L. 2012. Identification and detection of Phytophthora: Reviewing our progress, identifying our needs. Plant Dis. 96:1080-1103.

14. Park, J., Park, B., Veeraraghavan, N., Jung, K., Lee, Y.-H., Blair, J. E., Geiser, D. M., Isard, S., Mansfield, M., Nikolaeva, E., Park, S.-Y., Russo, J., Kim, S. H., Greene, M., Ivors, K. L., Balci, Y., Peiman, M., Erwin, D. C., Coffey, M. D., Rossman, A., Farr, D., Cline, E., Grünwald, N. J.,
Luster, D. G., Schrandt, J., Martin, F., Ribeiro, O. K., Makalowska, I., and Kang, S. 2008. Phytophthora database: A forensic database supporting the identification and monitoring of Phytophthora. Plant Dis. 92:966-972.

15. Prospero, S., Grünwald, N. J., Winton, L. M., and Hansen, E. M. 2009. Migration patterns of the emerging plant pathogen Phytophthora ramorum on the West Coast of the United States of America. Phytopathology 99:739-749.

16. Seifert, K. A. 2009. Progress towards DNA barcoding of fungi. Mol. Ecol. Resour. 9:83-89.

17. Vercauteren, A., Larsen, M., Goss, E., Grünwald, N. J., Maes, M., and Heungens, K. 2011. Identification of new polymorphic microsatellite markers in the NA1 and NA2 lineages of Phytophthora ramorum. Mycologia 103:1245-1249. 